\title{
Associations of serum 25-hydroxyvitamin D with metabolic syndrome and its components in elderly men and women: the Korean Urban Rural Elderly cohort study
}

Su Jin Lee ${ }^{1,2+}$, Eun Young Lee ${ }^{3+}$, Jung Hyun Lee ${ }^{2}$, Jong Eun Kim ${ }^{4}$, Kwang Joon Kim ${ }^{6}$, Yumie Rhee ${ }^{1}$, Hyeon Chang Kim², Yoosik Youm ${ }^{5}$ and Chang Oh Kim ${ }^{6 *}$

\begin{abstract}
Background: Many studies have investigated the association between vitamin D and metabolic syndrome (MetS). However, few studies have investigated the association stratified by sex in the elderly. Therefore, we aimed to evaluate the association between vitamin D, MetS, and its components in Korean elderly men and women.

Methods: A total of 987 men and 1949 women aged $\geq 65$ years were recruited through Korean Urban Rural Elderly cohort study. Serum 25-hydroxyvitamin D $(25(\mathrm{OH}) \mathrm{D})$ levels were categorized into 4 quartiles and all data were analyzed separately by sex. MetS was defined by the revised criteria of the National Cholesterol Education Program Adult Treatment Panel III.
\end{abstract}

Results: The participants in the lowest quartile of serum 25(OH)D showed a significant increase in the prevalence of high waist circumference, elevated triglyceride level, and low high-density lipoprotein cholesterol level, as well as MetS itself, in both men and women in a univariate analysis. After adjusting for potential confounders including age, smoking status, drinking status, exercise status, region of residence, seasonality, and parathyroid hormone level, the lowest 25(OH)D quartile group was associated with a higher risk of MetS (odds ratio [OR] 2.25, 95\% confidence interval [CI] 1.48-3.43 in men and OR 1.65, 95\% Cl 1.27-2.16 in women) compared to the highest 25(OH)D quartile group as the reference group. However, no significant association was found between serum 25(OH)D levels and the prevalence of MetS components including hyperglycemia or hypertension in both men and women.

Conclusions: Low 25(OH)D levels were associated with increased odds of MetS; in particular, they were associated with MetS components of high waist circumference, hypertriglyceridemia, and low high-density lipoproteincholesterol, after adjusting for age, smoking, alcohol, exercise, region of residency, and seasonality, in men and women over 65 years old.

Keywords: Vitamin D, Metabolic syndrome, Elderly, High waist circumference, Hypertriglyceridemia, Low highdensity lipoprotein

\footnotetext{
* Correspondence: COKIM@yuhs.ac

† Su Jin Lee and Eun Young Lee contributed equally to this work.

${ }^{6}$ Division of Geriatrics, Department of Internal Medicine, Yonsei University

College of Medicine, 50-1 Yonsei-ro, Seodaemun-gu, Seoul 03772, Republic

of Korea

Full list of author information is available at the end of the article
} 


\section{Background}

Vitamin D deficiency is a frequent public health issue in the elderly worldwide. In Korea, vitamin D deficiency was found in $47.3 \%$ of men and $64.5 \%$ females in 2008 [1]. More than 50\% of women and 30\% men had vitamin D levels below $20 \mathrm{ng} / \mathrm{mL}$ among individuals in their 60s and older populations in 2008 [1]. Vitamin D plays an important role in various physiological functions including maintaining calcium homeostasis and bone health. As vitamin $\mathrm{D}$ receptors are distributed in most of tissues like the muscle, pancreas, brain, prostate, and breast, among others, vitamin $\mathrm{D}$ is also involved in various extra-skeletal functions [2-4]. In the elderly, vitamin D deficiency is not only considered the risk factor of osteoporosis and fractures but also is associated with beta cell dysfunction and increased insulin resistance leading to cardiometabolic diseases such as diabetes and metabolic syndrome (MetS) [3-6].

MetS is a cluster of factors that have insulin resistant characteristics such as high waist circumference, high blood pressure, high blood sugar, and hyperlipidemia. MetS is one of the major public health concerns with increasing prevalence. The prevalence of MetS was 31.3\% in 2007 in Korea. Notably, the prevalence of MetS was the highest in individuals in their 60 s with as high as > $70 \%$ in women and approximately $50 \%$ in men [7]. Because vitamin D deficiency causes insulin resistance which is linked to MetS, the associations between vitamin D deficiency and MetS have been studied [8-10]. Many studies have revealed that vitamin D deficiency increases the prevalence of MetS in various populations. However, the results about the associations between the vitamin D level and each component of MetS in different populations are inconsistent $[9,11]$. Moreover, there were few studies focusing on the association of vitamin D level and MetS by sex-stratification over 65 years old. Thus, we aimed to elucidate the association of MetS and its components on the basis of vitamin D levels in elderly Korean men and women, separately.

\section{Methods}

\section{Study population and data collection}

We studied 2936 participants including 987 men and 1949 women aged $\geq 65$ years from the Korean Urban Rural Elderly cohort study [12]. Serum 25hydroxyvitamin D (25(OH)D) levels were categorized into 4 quartiles for both men and women. The revised criteria of the National Cholesterol Education Program Adult Treatment Panel III (NCEP-ATP III) were used to define MetS $[13,14]$. MetS was diagnosed if $\geq 3$ of the following criteria were met: (i) high waist circumference, defined as a waist circumference $\geq 90 \mathrm{~cm}$ for men or $\geq$ $80 \mathrm{~cm}$ for women; (ii) hypertriglyceridemia, defined as serum triglyceride level $\geq 150 \mathrm{mg} / \mathrm{dL}$; (iii) low highdensity lipoprotein (HDL) cholesterol, defined as a serum HDL cholesterol level $<40 \mathrm{mg} / \mathrm{dL}$ for men or $<50$ $\mathrm{mg} / \mathrm{dL}$ for women; (iv) hypertension, defined as a systolic blood pressure $\geq 130 \mathrm{mmHg}$, diastolic blood pressure $\geq 85 \mathrm{mmHg}$, or the use of an antihypertensive drug; (v) hyperglycemia, defined as fasting glucose $\geq 100 \mathrm{mg} /$ $\mathrm{dL}$ or the use of an antidiabetic drug. The study protocol was approved by the Ethics Committee of Severance Hospital (Seoul, Korea) (IRB No. 4-2012-0172).

\section{Biochemical measurements and questionnaires}

All blood samples were collected after an overnight fast (> 8-h fasting) and analyzed within $24 \mathrm{~h}$. Plasma glucose and creatinine levels were measured by performing a colorimetry immunoassay, and serum triglyceride, HDL cholesterol, and total cholesterol levels were also measured using an enzymatic method using an ADVIA 1800 Auto Analyzer (Siemens Medical Solutions, Malvern, PA, USA). Serum insulin level was measured by performing a chemiluminescence immunoassay using an ADVIA Centaur XP (Siemens Medical Solutions, Malvern, PA, USA). Serum 25(OH)D level was measured by using a chemiluminescence immunoassay (Liaison; DiaSorin, Dietzenbach, Germany). Intra- and interassay coefficients of variation ranged from 2.9 to $5.5 \%$ and from 6.5 to $12.9 \%$, respectively. Estimated glomerular filtration rate (eGFR) was calculated using the CKD-EPI [15]. Parathyroid hormone (PTH) level was measured by using an electrochemiluminescence immunoassay (E-170 modular, Roche, USA). Total coefficients of variation ranged from 2.8 to $3.4 \%$. Homeostatic model assessment-insulin resistance (HOMA-IR) was calculated by using the following equation: [fasting plasma insulin $(\mu \mathrm{IU} / \mathrm{mL}) \times$ fasting plasma glucose $(\mathrm{mg} / \mathrm{dL}) / 405$ ] [16]. At the first visit, self-reported questionnaires on patient medical histories, smoking status (current smoker or not), alcohol drinking status (current drinker or not), and exercise (currently involved in exercise or not) were administered.

\section{Statistical analysis}

All analyses were stratified by sex. Data were presented as mean \pm standard deviation or median [25th quartile - 75th quartile], or numbers (n) and percentages (\%). Analysis of variance (ANOVA) and Kruskal-Wallis tests were performed to compare groups on the basis of quartile of serum 25(OH)D level. Categorical variables were analyzed by using the $\chi^{2}$ or Mantel-Haenszel $\chi^{2}$ test. Trend test was performed for MetS and its components on the basis of the quartile of serum $25(\mathrm{OH}) \mathrm{D}$. Univariate regression analysis was used to evaluate the associations of serum 25(OH)D levels with MetS and its components 
including high waist circumference, hypertension, hyperglycemia, hypertriglyceridemia, and low HDL cholesterol, respectively. Multivariate logistic regression analyses were conducted to estimate the associations between vitamin $\mathrm{D}$ status and MetS, as well as between vitamin $\mathrm{D}$ status and each component of MetS. Model 1 was adjusted by age. Model 2 was adjusted by age, exercise status, and smoking status. Model 3 was adjusted by age, exercise status, smoking status, region of residence, and seasonality. Model 4 was adjusted by PTH level in addition to the covariates adjusted for in model 3. All statistical analyses were performed using SAS software, version 9.4 (SAS Institute Inc., Cary, NC, USA). A $p$-value of $<0.05$ was considered to be statistically significant.

\section{Results}

\section{Baseline characteristics}

The mean age of 2936 people in total was $71.9 \pm 4.6$ years $(72.8 \pm 4.6$ years in men and $71.5 \pm 4.6$ years in women, respectively). Overall, the mean $25(\mathrm{OH}) \mathrm{D}$ level was $18.0 \pm 7.8 \mathrm{ng} / \mathrm{mL}$. The level of serum $25(\mathrm{OH}) \mathrm{D}$ was higher in men than those in women $(19.7 \pm 7.5 \mathrm{ng} / \mathrm{mL}$ in men and $17.1 \pm 7.9 \mathrm{ng} / \mathrm{mL}$ in women, $p<0.0001)$. To evaluate the relationship between vitamin D levels and MetS, the study population was stratified according to the quartiles of serum 25(OH)D level for each sex. Clinical and biochemical characteristics of the participants divided into 4 groups stratified by sex are shown in Tables 1 and 2, respectively. For both sexes, waist circumference, PTH level, insulin level, and HOMA-IR, total cholesterol level, and triglyceride level were higher in the lowest $25(\mathrm{OH}) \mathrm{D}$ quartile, compared with the highest quartile of $25(\mathrm{OH}) \mathrm{D}$. There were no differences in age, body mass index, systolic and diastolic blood pressure, eGFR, and HDL cholesterol level among the 4 groups. The life style parameters such as smoking and drinking did not significantly differ among the 4 groups for both sexes. The number of MetS components was highest in the lowest $25(\mathrm{OH}) \mathrm{D}$ quartile and lowest in the highest 25(OH)D quartile in both sexes.

\section{Association of serum 25(OH)D levels with MetS and its components}

Among all participants, $45.4 \%$ of participants had MetS. The prevalence of MetS was higher in women (50.0\%) than in men (36.4\%). The prevalence of MetS according to quartile of vitamin D was presented in Fig. 1. As the $25(\mathrm{OH}) \mathrm{D}$ level decreased, the prevalence of MetS increased in both sexes ( $p$ for trend $<0.001$, both sexes) (Fig. 1). The prevalence of high waist circumference, hypertriglyceridemia, and low HDL cholesterol

Table 1 Baseline characteristics of men according to serum 25(OH)D level

\begin{tabular}{|c|c|c|c|c|c|}
\hline $\begin{array}{l}\text { Quartile (n) } \\
\text { (ng/mL) }\end{array}$ & $\begin{array}{l}\text { Quartile } 1(n=245) \\
(4.20-14.19)\end{array}$ & $\begin{array}{l}\text { Quartile 2 }(n=245) \\
(14.20-18.99)\end{array}$ & $\begin{array}{l}\text { Quartile } 3(n=249) \\
(19.00-24.19)\end{array}$ & $\begin{array}{l}\text { Quartile } 4(n=248) \\
(24.20-51.90)\end{array}$ & $P$ value \\
\hline $25(\mathrm{OH}) \mathrm{D}, \mathrm{ng} / \mathrm{mL}$ & $10.90 \pm 2.28$ & $16.53 \pm 1.39$ & $21.41 \pm 1.50$ & $29.67 \pm 5.34$ & $<0.001$ \\
\hline Age, yr & $73.01 \pm 4.87$ & $72.38 \pm 4.14$ & $72.59 \pm 4.47$ & $73.27 \pm 4.87$ & 0.132 \\
\hline $\mathrm{BMI}, \mathrm{kg} / \mathrm{m}^{2}$ & $23.99 \pm 2.63$ & $24.19 \pm 2.61$ & $23.65 \pm 2.67$ & $23.60 \pm 2.90 \dagger$ & 0.053 \\
\hline Waist, $\mathrm{cm}$ & $87.49 \pm 8.13$ & $86.68 \pm 8.44$ & $86.43 \pm 8.78$ & $83.48 \pm 9.33^{*}+\neq$ & $<0.001$ \\
\hline $\mathrm{SBP}, \mathrm{mmHg}$ & $125.89 \pm 14.50$ & $128.51 \pm 14.24$ & $129.70 \pm 14.41$ & $128.86 \pm 13.88$ & 0.370 \\
\hline $\mathrm{DBP}, \mathrm{mmHg}$ & $74.10 \pm 8.91$ & $73.68 \pm 9.28$ & $75.23 \pm 8.35$ & $73.02 \pm 7.90$ & 0.420 \\
\hline Current smoking & $42(17.1)$ & 53 (21.6) & $36(14.5)$ & $53(21.4)$ & 0.117 \\
\hline Current drinking & 205 (66.6) & $194(64.2)$ & $138(58)$ & $72(51.8)$ & 0.011 \\
\hline Exercise & $151(61.4)$ & $164(66.9)$ & $164(65.9)$ & $132(53.2)$ & 0.007 \\
\hline eGFR & $77.5 \pm 15.11$ & $79.8 \pm 14.03$ & $81.06 \pm 13.19^{*}$ & $80.13 \pm 13.44$ & 0.036 \\
\hline PTH & $39.68 \pm 14.13$ & $38.07 \pm 17.25$ & $35.80 \pm 12.07$ & $33.83 \pm 11.62 *+$ & $<0.001$ \\
\hline Glucose, mg/dL & $101.59 \pm 23.61$ & $100.47 \pm 20.17$ & $99.91 \pm 19.90$ & $97.50 \pm 19.93$ & 0.171 \\
\hline Insulin, IU/L & $7.53 \pm 8.31$ & $7.57 \pm 12.22$ & $6.12 \pm 4.26$ & $5.18 \pm 3.26^{*} \dagger$ & 0.001 \\
\hline HOMA-IR & $1.93 \pm 2.24$ & $1.86 \pm 2.42$ & $1.55 \pm 1.24$ & $1.29 \pm 0.99^{*} \dagger$ & $<0.001$ \\
\hline Total cholesterol, mg/dL & $174.51 \pm 32.91$ & $172.92 \pm 33.72$ & $173.93 \pm 33.09$ & $163.70 \pm 29.95^{*}+\ddagger$ & $<0.001$ \\
\hline Triglyceride, mg/dL & $138.69 \pm 78.55$ & $128.43 \pm 71.30$ & $125.45 \pm 61.22$ & $110.31 \pm 46.04^{*}+$ & $<0.001$ \\
\hline HDL cholesterol, mg/dL & $45.99 \pm 12.13$ & $47.89 \pm 12.56$ & $46.98 \pm 10.45$ & $48.15 \pm 11.26$ & 0.157 \\
\hline Number of MetS components & $2.22 \pm 1.30$ & $2.16 \pm 1.23$ & $2.02 \pm 1.25$ & $1.73 \pm 1.18^{*} \dagger$ & $<0.001$ \\
\hline
\end{tabular}

Data were shown as mean \pm SD or number (\%). 25(OH)D, vitamin D3, BMI body mass index, SBP systolic blood pressure, DBP diastolic blood pressure, eGFR estimated glomerular filtration rate, HOMA-IR homeostatic model assessment - insulin resistance, HDL high density lipoprotein, MetS metabolic syndrome. * vs. Q1, † vs. Q2, ‡ vs. Q3 
Table 2 Baseline characteristics of women according to serum 25(OH)D level

\begin{tabular}{|c|c|c|c|c|c|}
\hline $\begin{array}{l}\text { Quartile (n) } \\
\text { (ng/mL) }\end{array}$ & $\begin{array}{l}\text { Quartile 1 }(n=487) \\
(4.10-11.19)\end{array}$ & $\begin{array}{l}\text { Quartile 2 }(n=491) \\
(11.20-15.59)\end{array}$ & $\begin{array}{l}\text { Quartile 3 }(n=484) \\
(15.60-21.59)\end{array}$ & $\begin{array}{l}\text { Quartile } 4(n=487) \\
(21.60-54.90)\end{array}$ & $P$ value \\
\hline $25(\mathrm{OH}) \mathrm{D}, \mathrm{ng} / \mathrm{mL}$ & $8.72 \pm 1.67$ & $13.28 \pm 1.28$ & $18.31 \pm 1.70$ & $28.15 \pm 5.56$ & $<0.001$ \\
\hline Age, yr & $72.16 \pm 4.68$ & $71.07 \pm 4.71$ & $71.27 \pm 4.35$ & $71.45 \pm 4.54^{*} \dagger \neq$ & 0.001 \\
\hline BMl, $\mathrm{kg} / \mathrm{m}^{2}$ & $24.69 \pm 2.92$ & $25.07 \pm 3.31$ & $24.74 \pm 3.11$ & $23.58 \pm 2.85^{*} \dagger \neq$ & $<0.001$ \\
\hline Waist, cm & $83.49 \pm 8.71$ & $83.63 \pm 9.13$ & $83.14 \pm 8.52$ & $81.07 \pm 8.54^{*}+\ddagger$ & $<0.001$ \\
\hline $\mathrm{SBP}, \mathrm{mmHg}$ & $129.69 \pm 15.75$ & $128.51 \pm 16.42$ & $125.83 \pm 15.46$ & $124.69 \pm 15.21^{*}$ & 0.026 \\
\hline $\mathrm{DBP}, \mathrm{mmHg}$ & $74.15 \pm 7.96$ & $73.32 \pm 8.65$ & $72.55 \pm 8.75$ & $72.96 \pm 9.28$ & 0.451 \\
\hline Current smoking & $7(1.4)$ & $12(2.4)$ & $5(1)$ & $5(1)$ & 0.216 \\
\hline Current drinking & $99(23.2)$ & $91(20.6)$ & $145(28.4)$ & $137(24)$ & 0.043 \\
\hline Exercise & $293(60.2)$ & $308(62.7)$ & $294(60.7)$ & $294(60.4)$ & 0.855 \\
\hline eGFR & $81.04 \pm 15.49$ & $82.51 \pm 13.94$ & $82.74 \pm 13.23$ & $83.68 \pm 11.50^{*}$ & 0.024 \\
\hline PTH & $46.36 \pm 19.35$ & $41.12 \pm 14.40$ & $37.70 \pm 14.11$ & $36.28 \pm 13.80^{*}+$ & $<0.001$ \\
\hline Glucose, mg/dL & $96.11 \pm 16.34$ & $99.40 \pm 20.33$ & $98.17 \pm 19.57$ & $95.76 \pm 15.56 \dagger$ & 0.004 \\
\hline Insulin, IU/L & $7.63 \pm 4.79$ & $8.09 \pm 5.69$ & $7.60 \pm 5.07$ & $6.85 \pm 7.55+$ & 0.011 \\
\hline HOMA-IR & $1.85 \pm 1.35$ & $2.07 \pm 1.67$ & $1.89 \pm 1.47$ & $1.68 \pm 2.23 \dagger$ & 0.005 \\
\hline Total cholesterol, mg/dL & $190.70 \pm 38.79$ & $185.70 \pm 32.22$ & $187.60 \pm 36.56$ & $180.07 \pm 34.45^{*} \neq$ & $<0.001$ \\
\hline Triglyceride, mg/dL & $143.47 \pm 72.92$ & $127.91 \pm 61.37$ & $128.05 \pm 57.94$ & $115.83 \pm 51.41^{*}+\neq$ & $<0.001$ \\
\hline HDL cholesterol, mg/dL & $49.85 \pm 12.58$ & $51.63 \pm 12.61$ & $52.29 \pm 12.13$ & $52.30 \pm 12.12^{*}$ & 0.005 \\
\hline Number of MetS components & $2.65 \pm 1.29$ & $2.52 \pm 1.30$ & $2.50 \pm 1.32$ & $2.26 \pm 1.31^{*}+\neq$ & $<0.001$ \\
\hline
\end{tabular}

Data were shown as mean \pm SD or number (\%). $25(\mathrm{OH}) \mathrm{D}$, vitamin $\mathrm{D} 3, B M I$ body mass index, SBP systolic blood pressure, DBP diastolic blood pressure, eGFR estimated glomerular filtration rate, HOMA-IR homeostatic model assessment - insulin resistance, HDL high density lipoprotein, MetS metabolic syndrome. * vs. Q1, † vs. Q2, ‡ vs. Q3

significantly increased with decreasing vitamin D level; however, the prevalence of hyperglycemia showed this trend only in men ( $p$ for trend $=0.011$ ) (Table 3). Hypertension did not show a significant change with decreasing level of vitamin $\mathrm{D}$ in both sexes. On univariate analysis (Table 3), the odds of MetS significantly increased in the lowest quartile of 25(OH)D level (OR 2.22 [95\% CI, 1.52-2.34] in men and 1.98 [95\% CI, 1.60-2.44] in women). To control potential confounding factors that affect the outcome in addition to age; smoking, alcohol, and exercise statuses; seasonality, which could affect serum 25(OH)D level depending on the time when the examination was performed; and region of residence in which the participants live (e.g., rural or urban), were further adjusted in model 3 (Tables 4 and 5). The odds of MetS, high waist circumference, hypertriglyceridemia, and low HDL in the lower quartile groups were higher compared to those in the highest quartile group of $25(\mathrm{OH}) \mathrm{D}$ level in both men and women. With further adjustment for PTH in model 4, the pattern was similar; however, the association of high waist circumference in lowest quartile of serum $25(\mathrm{OH}) \mathrm{D}$ level disappeared in women (OR 1.28 [95\% CI, 0.97-1.68]), however, was persisted in men (OR 1.81 [95\% CI, 1.19-2.77.

Metabolic syndrome
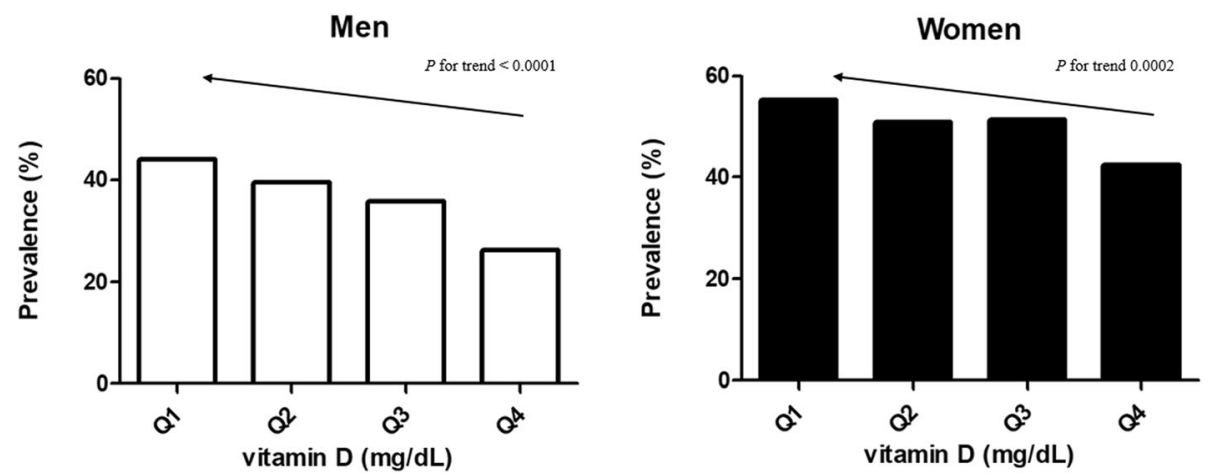

Fig. 1 The prevalence of metabolic syndrome and its components, on the basis of serum 25(OH)D level 
Table 3 Association of serum 25(OH)D levels with metabolic syndrome and its components in men and women

\begin{tabular}{|c|c|c|c|c|c|c|}
\hline $25(\mathrm{OH}) \mathrm{D}$ & Metabolic syndrome & Abdominal obesity & Hypertension & Hyperglycemia & Hypertriglyceridemia & low HDL \\
\hline Men & & & Univariate OR & & & \\
\hline Quartile 1 & $2.22(1.52-3.24)$ & $2.03(1.39-2.99)$ & $0.96(0.65-1.43)$ & $1.48(1.03-2.13)$ & $2.13(1.40-3.24)$ & $1.77(1.18-2.63)$ \\
\hline Quartile 2 & $1.85(1.26-2.70)$ & $1.93(1.32-2.84)$ & $1.05(0.70-1.56)$ & $1.72(1.20-2.47)$ & $1.78(1.16-2.74)$ & $1.35(0.90-2.03)$ \\
\hline Quartile 3 & $1.57(1.07-2.30)$ & $1.73(1.17-2.54)$ & $1.05(0.71-1.56)$ & $1.26(0.88-1.82)$ & $1.78(1.16-2.73)$ & $1.07(0.70-1.62)$ \\
\hline Quartile 4 & 1.00 (Reference) & 1.00 (Reference) & 1.00 (Reference) & 1.00 (Reference) & 1.00 (Reference) & 1.00 (Reference) \\
\hline$P$ for trend & $<0.001$ & $<0.001$ & 0.860 & 0.011 & 0.001 & 0.002 \\
\hline \multicolumn{7}{|c|}{ Women Univariate OR } \\
\hline Quartile 1 & $1.98(1.60-2.44)$ & $1.42(1.09-1.84)$ & $1.30(0.98-1.72)$ & $1.11(0.85-1.45)$ & $2.13(1.60-2.83)$ & $1.37(1.06-1.76)$ \\
\hline Quartile 2 & $1.40(1.09-1.81)$ & $1.35(1.04-1.74)$ & $1.20(0.91-1.58)$ & $1.30(1.00-1.69)$ & $1.43(1.07-1.92)$ & $1.12(0.87-1.44)$ \\
\hline Quartile 3 & $1.43(1.11-1.85)$ & $1.43(1.10-1.85)$ & $1.21(0.92-1.60)$ & $1.17(0.90-1.53)$ & $1.43(1.06-1.92)$ & $1.05(0.81-1.35)$ \\
\hline Quartile 4 & 1.00 (Reference) & 1.00 (Reference) & 1.00 (Reference) & 1.00 (Reference) & 1.00 (Reference) & 1.00 (Reference) \\
\hline$P$ for trend & $<0.001$ & 0.017 & 0.082 & 0.343 & 0.001 & 0.013 \\
\hline
\end{tabular}

25(OH)D: 25-OH-vitamin D, OR odds ratio, Cl confidence interval, $H D L$ high-density lipoprotein

\section{Discussion}

The relationship between vitamin D levels and MetS has been widely studied in several populations $[8,11,17$, 18]. However, most studies have not exclusively examined this association in elderly participants. With aging, cutaneous vitamin D production is reduced [4]. With aging, there are reductions in the renal production of $1,25(\mathrm{OH})_{2} \mathrm{D}$ by the kidneys, calcium absorption, and the action of vitamin D receptors [19]. Moreover, MetS is a concern throughout the aged society [20], as it is related to insulin resistance and obesity. Further, Vitamin D is lipid-soluble and is sequestered in fat tissue, which leads to lower vitamin D levels in obesity [21]. Thus, a specific relationship is expected between vitamin $\mathrm{D}$ levels and MetS prevalence in the elderly.

In this community-based cohort study involving Korean men and women aged $\geq 65$ years, we demonstrated that the risk of MetS was significantly higher in the lowest quartile compared to the highest quartile of the vitamin D level in both men and women. In particular, we found that a lower vitamin D level is significantly associated with a higher prevalence of high waist circumference, elevated triglyceride level, and low HDL level in both sexes independent of age, smoking status, alcohol drinking status, exercise status, region of residence, and seasonality.

Vitezova et al. revealed that higher $25(\mathrm{OH}) \mathrm{D}$ concentrations in the elderly were associated with lower prevalence of MetS and with higher HDL-cholesterol, lower triglyceride, lower waist circumference, and lower serum glucose [11]. However, in that study the results were combined for both men and women, and included both middle-aged and elderly adults; in contrast, in the present study, the results were stratified by sex, and the age of the study participants was $\geq 65$ years. Vitezova et al. [11] also reported a significant effect modification by sex $(P$ interactions $<0.05)$ in a subgroup analysis. They showed that a lower prevalence of high triglyceride level was significantly associated with high vitamin D level, similar to our findings.

In our study, MetS and its components, including hypertriglyceridemia and low-HDL level, were associated with vitamin $\mathrm{D}$ level in the lowest quartile group in both men and women in all models. However, the significance of the association between high waist circumference and vitamin $\mathrm{D}$ level in the lowest quartile group was maintained in men, but not in women. After additional adjustment by PTH level, the association between high waist circumference and vitamin D level was not maintained in men. Vitamin D deficiency causes elevation of PTH, which also affects the storage of fat [22]. Waist circumference is measured to reflect abdominal obesity; however, it does not completely represent the amount of visceral fat, which is more related to insulin resistance, and fat distribution, which could differ according to sex. Kim et al. [23] suggested sex-specific association of PTH and vitamin D with MetS. However, we did not observe a sex-specific effect on the association between vitamin $\mathrm{D}$ level and MetS, or between vitamin D level and specific MetS components. Bea et al. observed significant inverse relationships between $1,25(\mathrm{OH})_{2} \mathrm{D}$ and high triglyceride level; however, for $25(\mathrm{OH}) \mathrm{D}$ concentrations, significant inverse relationships were observed with MetS, waist circumference, and triglyceride [24]. In our sensitivity analysis for total population, 25(OH)D level as a continuous variable showed significant inverse relationships with MetS (OR 0.97, 95\% confidence interval $0.96-0.98$ ) by adjusting age, sex, smoking, alcohol, exercise, region of residence, seasonality, PTH. Oosterwerff et al. showed an increased risk of MetS in participants who were $>65$ 
Table 4 Multivariable-adjusted odds ratio of metabolic syndrome and its components according to serum 25(OH)D levels in men

\begin{tabular}{|c|c|c|c|c|c|c|c|}
\hline \multirow[t]{3}{*}{ Men } & \multicolumn{7}{|c|}{ Vitamin D level } \\
\hline & \multicolumn{2}{|c|}{ 1st quartile } & \multicolumn{2}{|c|}{ 2nd quartile } & \multicolumn{2}{|c|}{ 3rd quartile } & \multirow[t]{2}{*}{ 4th quartile } \\
\hline & $\overline{O R}$ & $\mathrm{Cl}$ & $\overline{O R}$ & $\mathrm{Cl}$ & $\overline{O R}$ & $\mathrm{Cl}$ & \\
\hline \multicolumn{8}{|c|}{ Metabolic syndrome } \\
\hline Model 1 & 2.22 & $(1.52-3.24)$ & 1.84 & $(1.26-2.70)$ & 1.56 & $(1.06-2.30)$ & \multirow[t]{4}{*}{1 (Reference) } \\
\hline Model 2 & 2.27 & $(1.55-3.33)$ & 1.87 & $(1.27-2.75)$ & 1.55 & $(1.05-2.28)$ & \\
\hline Model 3 & 2.03 & $(1.34-3.07)$ & 1.70 & $(1.14-2.55)$ & 1.45 & $(0.98-2.16)$ & \\
\hline Model 4 & 2.25 & $(1.48-3.43)$ & 1.83 & $(1.22-2.74)$ & 1.51 & $(1.02-2.24)$ & \\
\hline \multicolumn{8}{|c|}{ Abdominal obesity } \\
\hline Model 1 & 2.03 & $(1.38-2.98)$ & 1.92 & $(1.31-2.83)$ & 1.72 & $(1.17-2.53)$ & \multirow[t]{4}{*}{1} \\
\hline Model 2 & 2.10 & $(1.43-3.10)$ & 1.99 & $(1.35-2.93)$ & 1.73 & $(1.17-2.56)$ & \\
\hline Model 3 & 1.81 & $(1.19-2.75)$ & 1.79 & $(1.19-2.68)$ & 1.64 & $(1.10-2.44)$ & \\
\hline Model 4 & 1.81 & $(1.19-2.77)$ & 1.79 & $(1.19-2.69)$ & 1.64 & $(1.10-2.45)$ & \\
\hline \multicolumn{8}{|c|}{ Hypertension } \\
\hline Model 1 & 0.97 & $(0.65-1.44)$ & 1.07 & $(0.72-1.60)$ & 1.07 & $(0.72-1.59)$ & \multirow[t]{4}{*}{1} \\
\hline Model 2 & 1.03 & $(0.69-1.55)$ & 1.10 & $(0.73-1.65)$ & 1.05 & $(0.70-1.57)$ & \\
\hline Model 3 & 1.05 & $(0.68-1.62)$ & 1.10 & $(0.72-1.69)$ & 1.04 & $(0.69-1.57)$ & \\
\hline Model 4 & 1.07 & $(0.69-1.67)$ & 1.12 & $(0.73-1.72)$ & 1.05 & $(0.69-1.59)$ & \\
\hline \multicolumn{8}{|c|}{ Hyperglycemia } \\
\hline Model 1 & 1.48 & $(1.03-2.13)$ & 1.72 & $(1.20-2.47)$ & 1.26 & $(0.88-1.82)$ & \multirow[t]{4}{*}{1} \\
\hline Model 2 & 1.49 & $(1.03-2.14)$ & 1.69 & $(1.17-2.44)$ & 1.22 & $(0.85-1.76)$ & \\
\hline Model 3 & 1.18 & $(0.79-1.75)$ & 1.42 & $(0.97-2.09)$ & 1.10 & $(0.75-1.60)$ & \\
\hline Model 4 & 1.34 & $(0.90-2.01)$ & 1.57 & $(1.06-2.31)$ & 1.15 & $(0.79-1.68)$ & \\
\hline \multicolumn{8}{|c|}{ Hypertriglycemia } \\
\hline Model 1 & 2.11 & $(1.38-3.23)$ & 1.74 & $(1.13-2.67)$ & 1.74 & $(1.14-2.68)$ & \multirow[t]{4}{*}{1} \\
\hline Model 2 & 2.27 & $(1.47-3.48)$ & 1.79 & $(1.16-2.76)$ & 1.83 & $(1.19-2.82)$ & \\
\hline Model 3 & 2.41 & $(1.51-3.86)$ & 1.87 & $(1.19-2.96)$ & 1.89 & $(1.21-2.94)$ & \\
\hline Model 4 & 2.77 & $(1.72-4.48)$ & 2.05 & $(1.29-3.25)$ & 1.99 & $(1.28-3.11)$ & \\
\hline \multicolumn{8}{|l|}{ Low HDL } \\
\hline Model 1 & 1.76 & $(1.18-2.63)$ & 1.34 & $(0.89-2.02)$ & 1.06 & $(0.70-1.62)$ & \multirow[t]{4}{*}{1} \\
\hline Model 2 & 1.61 & $(1.07-2.41)$ & 1.30 & $(0.86-1.97)$ & 1.04 & $(0.68-1.59)$ & \\
\hline Model 3 & 1.55 & $(1.00-2.41)$ & 1.28 & $(0.83-1.98)$ & 1.04 & $(0.67-1.61)$ & \\
\hline Model 4 & 1.70 & $(1.09-2.67)$ & 1.36 & $(0.88-2.12)$ & 1.07 & $(0.69-1.66)$ & \\
\hline
\end{tabular}

Model 1: age adjusted, Model 2: Model $1+$ smoking, alcohol, exercise adjusted, Model 3: Model $2+$ region, seasonality adjusted, Model 4: Model $3+$ parathyroid hormone adjusted, $\mathrm{OR}$ odds ratio, $\mathrm{Cl}$ confidence interval, $\mathrm{HDL}$ high density lipoprotein

years of age with vitamin $\mathrm{D}$ level $<50 \mathrm{nmol}$ in the Netherlands [25]. They showed that low HDL cholesterol levels and high waist circumference were related to vitamin $\mathrm{D}$ deficiency. In that study, both sexes were analyzed together. When we analyzed both sexes together, the risk of MetS and its components including high waist circumference, hypertriglyceridemia and low HDL were significantly higher in the lowest quartile compared to the highest quartile of the vitamin D level. An additional file 1 shows this in more detail (see Additional file 1). Compared with the other studies, a possible explanation for the distinct findings of our results may comprise differences in the ages of participants in the studies. The differences in results regarding the associations between vitamin D level and MetS components in young and middle-aged populations stratified on the basis of sex may be influenced by hormonal levels $[10,26]$. In our study, no significant associations were observed between vitamin D levels and hypertension or hyperglycemia, which may have occurred because the participants included individuals already taking antihypertensive or antidiabetic drugs.

This study has certain limitations. First, this was a cross-sectional study. A meta-analysis has shown that while the correlation between serum vitamin D status and MetS 
Table 5 Multivariable-adjusted odds ratio of metabolic syndrome and its components according to serum 25(OH)D levels in women

\begin{tabular}{|c|c|c|c|c|c|c|c|}
\hline \multirow[t]{3}{*}{ Women } & \multicolumn{7}{|c|}{ Vitamin D level } \\
\hline & \multicolumn{2}{|c|}{ 1st quartile } & \multicolumn{2}{|c|}{ 2nd quartile } & \multicolumn{2}{|c|}{ 3rd quartile } & \multirow[t]{2}{*}{ 4th quartile } \\
\hline & $\mathrm{OR}$ & $\mathrm{Cl}$ & $\mathrm{OR}$ & $\mathrm{Cl}$ & $\mathrm{OR}$ & $\mathrm{Cl}$ & \\
\hline \multicolumn{8}{|c|}{ Metabolic syndrome } \\
\hline Model 1 & 1.63 & $(1.27-2.11)$ & 1.43 & $(1.11-1.84)$ & 1.45 & $(1.12-1.86)$ & \multirow[t]{4}{*}{1 (reference) } \\
\hline Model 2 & 1.35 & $(1.27-2.12)$ & 1.45 & $(1.12-1.86)$ & 1.45 & $(1.12-1.87)$ & \\
\hline Model 3 & 1.61 & $(1.24-2.09)$ & 1.42 & $(1.10-1.84)$ & 1.43 & $(1.11-1.84)$ & \\
\hline Model 4 & 1.65 & $(1.27-2.16)$ & 1.44 & $(1.12-1.87)$ & 1.43 & $(1.11-1.85)$ & \\
\hline \multicolumn{8}{|c|}{ Abdominal obesity } \\
\hline Model 1 & 1.39 & $(1.07-1.81)$ & 1.36 & $(1.05-1.76)$ & 1.44 & $(1.11-1.86)$ & \multirow[t]{4}{*}{1} \\
\hline Model 2 & 1.39 & $(1.08-1.81)$ & 1.36 & $(1.05-1.77)$ & 1.44 & $(1.11-1.87)$ & \\
\hline Model 3 & 1.33 & $(1.02-1.74)$ & 1.33 & $(1.03-1.73)$ & 1.42 & $(1.09-1.84)$ & \\
\hline Model 4 & 1.28 & $(0.97-1.68)$ & 1.30 & $(1.00-1.70)$ & 1.41 & $(1.08-1.83)$ & \\
\hline \multicolumn{8}{|c|}{ Hypertension } \\
\hline Model 1 & 1.24 & $(0.94-1.65)$ & 1.24 & $(0.94-1.64)$ & 1.23 & $(0.93-1.63)$ & \multirow[t]{4}{*}{1} \\
\hline Model 2 & 1.24 & $(0.94-1.65)$ & 1.24 & $(0.94-1.64)$ & 1.23 & $(0.93-1.63)$ & \\
\hline Model 3 & 1.30 & $(0.97-1.73)$ & 1.27 & $(0.96-1.68)$ & 1.25 & $(0.94-1.66)$ & \\
\hline Model 4 & 1.24 & $(0.92-1.66)$ & 1.24 & $(0.93-1.64)$ & 1.24 & $(0.93-1.64)$ & \\
\hline \multicolumn{8}{|c|}{ Hyperglycemia } \\
\hline Model 1 & 1.09 & $(0.83-1.42)$ & 1.31 & $(1.01-1.71)$ & 1.18 & $(0.90-1.54)$ & \multirow[t]{4}{*}{1} \\
\hline Model 2 & 1.08 & $(0.83-1.42)$ & 1.31 & $(1.00-1.70)$ & 1.18 & $(0.90-1.54)$ & \\
\hline Model 3 & 1.05 & $(0.80-1.38)$ & 1.28 & $(0.98-1.67)$ & 1.15 & $(0.88-1.51)$ & \\
\hline Model 4 & 1.14 & $(0.86-1.51)$ & 1.33 & $(1.02-1.75)$ & 1.17 & $(0.89-1.53)$ & \\
\hline \multicolumn{8}{|c|}{ Hypertriglycemia } \\
\hline Model 1 & 2.14 & $(1.61-2.85)$ & 1.42 & $(1.06-1.91)$ & 1.43 & $(1.06-1.91)$ & \multirow[t]{4}{*}{1} \\
\hline Model 2 & 2.16 & $(1.62-2.88)$ & 1.44 & $(1.07-1.44)$ & 1.43 & $(1.06-1.92)$ & \\
\hline Model 3 & 2.12 & $(1.59-2.84)$ & 1.44 & $(1.07-1.93)$ & 1.43 & $(1.06-1.93)$ & \\
\hline Model 4 & 2.14 & $(1.59-2.89)$ & 1.44 & $(1.07-1.95)$ & 1.43 & $(1.07-1.93)$ & \\
\hline \multicolumn{8}{|l|}{ Low HDL } \\
\hline Model 1 & 1.35 & $(1.05-1.73)$ & 1.13 & $(0.88-1.46)$ & 1.05 & $(0.82-1.35)$ & \multirow[t]{4}{*}{1} \\
\hline Model 2 & 1.36 & $(1.05-1.75)$ & 1.15 & $(0.90-1.49)$ & 1.05 & $(0.82-1.35)$ & \\
\hline Model 3 & 1.38 & $(1.07-1.79)$ & 1.17 & $(0.90-1.50)$ & 1.06 & $(0.82-1.37)$ & \\
\hline Model 4 & 1.39 & $(1.07-1.81)$ & 1.17 & $(0.91-1.51)$ & 1.06 & $(0.82-1.37)$ & \\
\hline
\end{tabular}

Model 1: age adjusted, Model 2: Model $1+$ smoking, alcohol, exercise adjusted, Model 3: Model 2 + region, seasonality adjusted, Model 4: Model 3 + parathyroid hormone adjusted, $O R$ odds ratio, $C l$ confidence interval, $H D L$ high density lipoprotein

prevalence in the general adult population has been established in cross-sectional studies, a significant relationship has not been reproduced in longitudinal studies [8]. However, in that meta-analysis the study populations of the included studies were heterogenous. The present cohort is scheduled to be followed-up. Thus, the longitudinal data obtained will be analyzed to explore the association between the change in vitamin $\mathrm{D}$ status and incidence of MetS in this population. Second, we could not consider factors such as calcium and vitamin D intake, which could have affected the serum levels of vitamin D. Nonetheless, the present study also has important strengths. Most previous studies included adults with age $>20$ years or women in their 50 s during the early-postmenopausal period. However, our study exclusively focused on the elderly Korean participants aged $\geq 65$ years and our analyses were also stratified by sex. Thus, the effects of the vitamin D level were examined with little or no hormonal effect.

\section{Conclusions}

In conclusion, vitamin D deficiency is associated with a higher prevalence of MetS in Korean elderly men and women. Adequate vitamin D level is important to decrease the risk of MetS. Further studies should evaluate the effect of vitamin D supplements on MetS prevalence in the elderly. 


\section{Additional file}

Additional file 1: Multivariable-adjusted odds ratio of metabolic syndrome and its components according to serum 25(OH)D levels stratified by body mass index (BMI). (DOCX $22 \mathrm{~kb}$ )

\section{Abbreviations}

25(OH)D: 25-hydroxyvitamin D; HDL: High-density lipoprotein; HOMAIR: Homeostatic model assessment-insulin resistance; MetS: Metabolic syndrom; NCEP-ATP III: National Cholesterol Education Program Adult Treatment Panel III; PTH: Parathyroid hormone

\section{Acknowledgements}

The authors thank Dae In Kim (Librarian, Yonsei University Medical Library) for his assistance in providing formatting the script.

\section{Funding}

This research was supported by funding (2011-E63005-00, 2012-E63001-001, 2013-E63007-00, 2013-E63007-01, 2013-E63007-02) from the Research of Korea Centers for Disease Control and Prevention.

\section{Availability of data and materials}

The data are available from the corresponding author on reasonable request.

\section{Authors' contributions}

Study conduct: SJL, EYL, JHL, JEK, KJK, HCK, YY, YR, COK. Data collection and interpretation: SJL, EYL, JHL, JEK, HCK, YR, COK. Statistical analysis: SJL, EYL, $J H L$, KJK. Drafting of the manuscript: SJL and EYL. COK takes responsibility for the integrity of the data analysis. All authors read and approved the final version of the manuscript.

\section{Ethics approval and consent to participate}

Written informed consent was obtained from all participants. The study protocol was approved by the Ethics Committee of Severance Hospital (IRB No. 4-2012-0172), and the study was conducted in accordance with the ethical standards of the Declaration of Helsinki.

\section{Consent for publication}

Not applicable.

\section{Competing interests}

The authors declare that they have no competing interests.

\section{Publisher's Note}

Springer Nature remains neutral with regard to jurisdictional claims in published maps and institutional affiliations.

\section{Author details \\ ${ }^{1}$ Department of Internal Medicine, Severance Hospital, Endocrine Research Institute, Yonsei University College of Medicine, Seoul, South Korea. ${ }^{2}$ Department of Preventive Medicine, Yonsei University College of Medicine, 50-1 Yonsei-ro, Seodaemun-gu, Seoul, South Korea. ${ }^{3}$ Division of Endocrinology and Metabolism, Department of Internal Medicine, Seoul St. Mary's Hospital, College of Medicine, The Catholic University of Korea, Seoul, South Korea. ${ }^{4}$ Graduate School of Public Health Yonsei University, Seoul, South Korea. ${ }^{5}$ Department of Sociology, Yonsei University, 50 Yonsei-ro, Seodaemun-gu, Seoul, South Korea. ${ }^{6}$ Division of Geriatrics, Department of Internal Medicine, Yonsei University College of Medicine, 50-1 Yonsei-ro, Seodaemun-gu, Seoul 03772, Republic of Korea.}

Received: 23 August 2018 Accepted: 25 March 2019 Published online: 11 April 2019

\section{References}

1. Choi HS, Oh HJ, Choi H, Choi WH, Kim JG, Kim KM, Kim KJ, Rhee Y, Lim S-K. Vitamin $D$ insufficiency in Korea - a greater threat to younger generation: the Korea National Health and nutrition examination survey (KNHANES) 2008. J Clin Endocrinol Metab. 2011;96(3):643-51.
2. Albrechtsson E, Jonsson T, Möller S, Höglund M, Ohlsson B, Axelson J. Vitamin D receptor is expressed in pancreatic cancer cells and a vitamin D3 analogue decreases cell number. Pancreatology. 2003;3(1):41-6.

3. Holick MF. Vitamin D deficiency. N Engl J Med. 2007;357(3):266-81.

4. Wacker M, Holick MF. Vitamin D - effects on skeletal and extraskeletal health and the need for supplementation. Nutrients. 2013;5(1):111-48.

5. Chiu KC, Chu A, Go VLW, Saad MF. Hypovitaminosis D is associated with insulin resistance and $\beta$ cell dysfunction. Am J Clin Nutr. 2004;79(5):820-5.

6. Boucher BJ. The problems of vitamin d insufficiency in older people. Aging Dis. 2012;3(4):313-29.

7. Lim S, Shin H, Song JH, Kwak SH, Kang SM, Won Yoon J, Choi SH, Cho SI, Park KS, Lee HK, et al. Increasing prevalence of metabolic syndrome in Korea: the Korean National Health and nutrition examination survey for 1998-2007. Diabetes Care. 2011;34(6):1323-8.

8. Ju SY, Jeong HS, Kim DH. Blood vitamin D status and metabolic syndrome in the general adult population: a dose-response meta-analysis. J Clin Endocrinol Metab. 2014;99(3):1053-63.

9. Chon SJ, Yun BH, Jung YS, Cho SH, Choi YS, Kim SY, Lee BS, Seo SK. Association between vitamin D status and risk of metabolic syndrome among Korean postmenopausal women. PLoS One. 2014;9(2):e89721.

10. Jorde R, Grimnes $G$. Vitamin D and metabolic health with special reference to the effect of vitamin D on serum lipids. Prog Lipid Res. 2011;50(4):303-12.

11. Vitezova A, Zillikens MC, van Herpt TT, Sijbrands EJ, Hofman A, Uitterlinden AG, Franco OH, Kiefte-de Jong JC. Vitamin D status and metabolic syndrome in the elderly: the Rotterdam study. Eur J Endocrinol. 2015;172(3): 327-35.

12. Lee EY, Kim HC, Rhee Y, Youm Y, Kim KM, Lee JM, Choi DP, Yun YM, Kim CO. The Korean urban rural elderly cohort study: study design and protocol. BMC Geriatr. 2014;14(1):33.

13. Third Report of the National Cholesterol Education Program (NCEP) Expert Panel on Detection, Evaluation, and Treatment of High Blood Cholesterol in Adults (Adult Treatment Panel III) final report. Circulation. 2002;106(25): 3143-421.

14. Grundy SM, Brewer HB Jr, Cleeman Jl, Smith SC Jr, Lenfant C. Definition of metabolic syndrome: report of the National Heart, Lung, and Blood Institute/American Heart Association conference on scientific issues related to definition. Circulation. 2004;109(3):433-8.

15. Levey AS, Stevens LA, Schmid CH, Zhang YL, Castro AF 3rd, Feldman HI, Kusek JW, Eggers $P$, Van Lente $F$, Greene $T$, et al. A new equation to estimate glomerular filtration rate. Ann Intern Med. 2009;150(9):604-12.

16. Wallace TM, Levy JC, Matthews DR. Use and abuse of HOMA modeling. Diabetes Care. 2004:27(6):1487-95.

17. Reis JP, von Mühlen D, Kritz-Silverstein D, Wingard DL, Barrett-Connor E. Vitamin $D$, parathyroid hormone levels, and the prevalence of metabolic syndrome in community-dwelling older adults. Diabetes Care. 2007;30(6):1549-55.

18. Gagnon C, Lu ZX, Magliano DJ, Dunstan DW, Shaw JE, Zimmet PZ, Sikaris K, Ebeling PR, Daly RM. Low serum 25-hydroxyvitamin D is associated with increased risk of the development of the metabolic syndrome at five years: results from a national, population-based prospective study (the Australian diabetes, obesity and lifestyle study: AusDiab). J Clin Endocrinol Metab. 2012;97(6):1953-61.

19. Gallagher JC. Vitamin D and Aging. Endocrinol Metab Clin N Am. 2013;42(2): 319-32.

20. Morley JE. The metabolic syndrome and aging. J Gerontol A Biol Sci Med Sci. 2004;59(2):139-42

21. Vanlint S. Vitamin D and obesity. Nutrients. 2013;5(3):949-56.

22. Zemel MB. Role of dietary calcium and dairy products in modulating adiposity. Lipids. 2003;38(2):139-46.

23. Kim MH, Lee J, Ha J, Jo K, Lim DJ, Lee JM, Chang SA, Kang MI, Cha BY. Gender specific association of parathyroid hormone and vitamin D with metabolic syndrome in population with preserved renal function. Sci Rep. 2018;8(1):1149.

24. Bea JW, Jurutka PW, Hibler EA, Lance P, Martinez ME, Roe DJ, Sardo Molmenti CL, Thompson PA, Jacobs ET. Concentrations of the vitamin D metabolite 1,25(OH)2D and odds of metabolic syndrome and its components. Metabolism. 2015;64(3):447-59.

25. Oosterwerff MM, Eekhoff EMW, Heymans MW, Lips P, van Schoor NM. Serum 25-hydroxyvitamin D levels and the metabolic syndrome in older persons: a population-based study. Clin Endocrinol. 2011;75(5):608-13.

26. Cooke PS, Naaz A. Role of estrogens in adipocyte development and function. Exp Biol Med (Maywood). 2004;229(11):1127-35. 